\title{
DERIVATIVES OF THE DIAMETER AND THE AREA OF A CONNECTED COMPONENT OF THE PSEUDOSPECTRUM*
}

\author{
GORKA ARMENTIA ${ }^{\dagger}$, JUAN-MIGUEL GRACIA $^{\ddagger}$, AND FRANCISCO E. VELASCO ${ }^{\ddagger}$
}

Abstract. The paper concerns the relation between the following two quantities.

(i) The Hölder condition number of an eigenvalue $\lambda$ of a square complex matrix.

(ii) The rate of growth of the diameter and the area of the connected component of the $\varepsilon$-pseusospectrum containing $\lambda$.

Key words. Matrices, Pseudospectra, Condition number, Eigenvalues, Derivatives.

AMS subject classifications. 15A12, 15A18, 15A60, 65F35.

1. Introduction. By $\Lambda(A)$ we denote the spectrum of any $A \in \mathbb{C}^{n \times n}$. We denote by $\|\cdot\|$ the 2-norm. Let $\lambda$ be an eigenvalue of $A$ of algebraic multiplicity $m$. For $X \in \mathbb{C}^{n \times n}, \operatorname{sv}_{(A, \lambda)}(X)$ denotes the radius of the smallest circle centered at $\lambda$ containing $m$ of the eigenvalues of $X$ counting multiplicities. The (Hölder) condition number of the eigenvalue $\lambda$ of order $\omega>0$ is defined as

$$
\operatorname{cond}_{\omega}(A, \lambda):=\lim _{\varepsilon \rightarrow 0^{+}} \max _{0<\|X-A\| \leq \varepsilon} \frac{\operatorname{sv}_{(A, \lambda)}(X)}{\|X-A\|^{\omega}} .
$$

The index of an eigenvalue $\lambda$ of $A, \nu=\nu(\lambda)$, is the size of the largest Jordan block associated with $\lambda$. The limit that defines $\operatorname{cond}_{\omega}(A, \lambda)$ is of interest just for $\omega=1 / \nu$.

On the other hand, for $\varepsilon \geq 0$, the $\varepsilon$-pseudospectrum of $A$ consists of the eigenvalues of all matrices $X$ within an $\varepsilon$-neighborhood of $A$, i.e.,

$$
\Lambda_{\varepsilon}(A):=\bigcup_{\substack{X \in \mathbb{C}^{n \times n} \\\|X-A\| \leq \varepsilon}} \Lambda(X) .
$$

${ }^{*}$ Received by the editors on November 15, 2010. Accepted for publication on September 11, 2011. Handling Editor: Michael Tsatsomeros. This paper has been written financed by the MTM201019356-C02-01 project of the MICINN Spanish Ministry, and the GIC10/169-IT361-10 aid of the Basque Government.

${ }^{\dagger}$ Department of Mathematical Engineering and Computer Science, The Public University of Navarre, Campus de Arrosadía, 31006 Pamplona, Spain (gorka.armentia@unavarra.es).

${ }^{\ddagger}$ Department of Applied Mathematics and Statistics and R.O., The University of the Basque Country, Faculty of Pharmacy, Paseo de la Universidad 7, 01006 Vitoria-Gasteiz, Spain (juanmiguel.gracia@ehu.es, franciscoenrique.velasco@ehu.es). 
For any complex matrix $M$ we denote by $\sigma_{1}(M) \geq \sigma_{2}(M) \geq \cdots$ its singular values arranged in decreasing order. It is well known that

$$
\Lambda_{\varepsilon}(A)=\left\{z \in \mathbb{C}: \sigma_{n}(z I-A) \leq \varepsilon\right\} .
$$

The subset $\Lambda_{\varepsilon}(A)$ of the complex plane is a compact set consisting of at most $r$ (disjoint) connected components, one around each eigenvalue, where $\Lambda(A)=\left\{\lambda_{1}, \ldots, \lambda_{r}\right\}$. Denote the connected component of $\Lambda_{\varepsilon}(A)$ around the eigenvalue $\lambda$ by $\mathcal{K}_{\lambda}(\varepsilon)$, and consider the diameter $\delta(\varepsilon)$ and the area $a(\varepsilon)$ of this component as a function of $\varepsilon$.

We denote by $\partial \mathcal{K}_{\lambda}(\varepsilon)$ the boundary of $\mathcal{K}_{\lambda}(\varepsilon)$. From [6, Proposition 2.6.5], we deduce that

$$
c=\lim _{\varepsilon \rightarrow 0^{+}} \frac{\max _{z \in \partial \mathcal{K}_{\lambda}(\varepsilon)}|z-\lambda|}{\varepsilon^{1 / \nu}},
$$

where $c$ denotes the Hölder condition number $\operatorname{cond}_{1 / \nu}(A, \lambda)$. Thus, calling $\rho(\varepsilon):=$ $\max _{z \in \partial \mathcal{K}_{\lambda}(\varepsilon)}|z-\lambda|$, we see that $\lim _{\varepsilon \rightarrow 0^{+}} \frac{\rho(\varepsilon)}{\varepsilon^{1 / \nu}}=c$. We extend this result to $\delta(\varepsilon)$ and $a(\varepsilon)$ instead of $\rho(\varepsilon)$ in Theorems 4.1 and 6.1 .

REMARK 1.1. When $\lim _{\varepsilon \rightarrow 0^{+}} \frac{\delta(\varepsilon)}{\varepsilon}=\infty$ (resp., $\left.\lim _{\varepsilon \rightarrow 0^{+}} \frac{a(\varepsilon)}{\varepsilon}=\infty\right)$, strictly speaking the function $\delta(\varepsilon)$ (resp., $a(\varepsilon)$ ) is not differentiable from the right-hand side at 0 . However, in this case we put $\delta_{+}^{\prime}(0)=\infty$ (resp., $a_{+}^{\prime}(0)=\infty$ ) in order to grasp the geometric meaning of the results.

The main results of the paper are as follows.

1. $\delta_{+}^{\prime}(0)=2 c$ if $\nu=1$. Otherwise $\delta_{+}^{\prime}(0)=\infty$. Here, $\delta_{+}^{\prime}$ denotes the rightderivative of $\delta$. See Theorem 5.2.

2. $a_{+}^{\prime}(0)=0$ if $\nu=1$, and $a_{+}^{\prime}(0)=\pi c^{2}$ if $\nu=2$. Otherwise, $a_{+}^{\prime}(0)=\infty$. See Theorem 7.2.

In Section 2, we work four examples of matrices $A$ for which both the condition number and the geometry of the $\varepsilon$-pseudospectrum are known in detail; this let us corroborate our results. We demonstrate that an important result by Karow [6] allows us bound the $\varepsilon$-pseudospectrum by lower and upper closed disks; see Section 3. By the monotonicity of the diameter function, and its changes under a homothecy, we show how to transfer these inequalities with respect to the inclusion relation between sets to the diameters, in Section 4. In Section 5, we relate the first right-derivative of the diameter at $\varepsilon=0$ with the condition number. In Section 6 , the monotonicity of the area function, and its changes under a homothecy, let us translate the bounds in Section 3 to numeric inequalities. In Section 7, we relate the first right-derivative of the area at $\varepsilon=0$ with the condition number $c$; moreover, we establish a relation between $c$ and the second right-derivative $a_{+}^{\prime \prime}(0)$ whenever this derivative exists. Finally, in 
Section 8 , we formulate a conjecture about the semialgebraicity of the functions $\delta(\varepsilon)$ and $a(\varepsilon)$; if it were true, a de l'Hôpital inverse rule would let us prove the existence of $a_{+}^{\prime \prime}(0)$.

2. Examples. Next, we consider four examples where we compute the condition number of order $1 / \nu$ of an eigenvalue $\lambda$ and the right-derivatives at $\varepsilon=0$ of the diameter $\delta(\varepsilon)$ and the area $a(\varepsilon)$ of the connected component $\mathcal{K}_{\lambda}(\varepsilon)$.

EXAMPLE 2.1. Let $A \in \mathbb{C}^{n \times n}$ be a normal matrix. Then the $\varepsilon$-pseudospectrum of $A$ is the union of the closed disks of radius $\varepsilon$ centered at the eigenvalues of $A$. So, for sufficiently small $\varepsilon \geq 0$, we have

$$
\mathcal{K}_{\lambda}(\varepsilon)=\mathcal{D}(\lambda, \varepsilon)
$$

Therefore, $\rho(\varepsilon)=\varepsilon$, and since the eigenvalues of a normal matrix are semisimple (i.e., of index 1),

$$
\operatorname{cond}_{1}(A, \lambda)=\lim _{\varepsilon \rightarrow 0^{+}} \frac{\varepsilon}{\varepsilon}=1 \text {. }
$$

The diameter of $\mathcal{D}(\lambda, \varepsilon)$ is $2 \varepsilon$. So, $\delta^{\prime}(\varepsilon)=2$ and $\delta_{+}^{\prime}(0)=2$. If we denote by $a(\varepsilon)$ the area of this circle, $a(\varepsilon)=\pi \varepsilon^{2}$; hence, $a^{\prime}(\varepsilon)=2 \pi \varepsilon, a^{\prime \prime}(\varepsilon)=2 \pi$. Therefore, $a_{+}^{\prime}(0)=0, a_{+}^{\prime \prime}(0)=2 \pi$.

EXAMPLE 2.2. Let

$$
J_{2}(\lambda, d)=\left[\begin{array}{ll}
\lambda & d \\
0 & \lambda
\end{array}\right]
$$

be like a Jordan block, with complex numbers $\lambda, d$ and $d \neq 0$. Karow proved in $[6$, Theorem 5.4.1, p. 74] that for each $\varepsilon \geq 0$,

$$
\Lambda_{\varepsilon}\left(J_{2}(\lambda, d)\right)
$$

is a closed disk centered at $\lambda$ and with radius $r_{2}(\varepsilon)=\max \left\{r>0 \mid \sigma_{2}\left(J_{2}(r,|d|)\right) \leq \varepsilon\right\}$. As

$$
\sigma_{2}\left(J_{2}(r,|d|)\right)=\sqrt{r^{2}+\frac{|d|^{2}}{2}-\sqrt{r^{2}|d|^{2}+\frac{|d|^{4}}{4}}},
$$

solving the equation in the unknown $r$

$$
r^{2}+\frac{|d|^{2}}{2}-\sqrt{r^{2}|d|^{2}+\frac{|d|^{4}}{4}}=\varepsilon^{2},
$$

we find that $r_{2}(\varepsilon)=\sqrt{\varepsilon^{2}+|d|}$. This result has also been proved by Cui et al. [4, Proposition 2.1]. So, $\delta(\varepsilon)=2 \sqrt{\varepsilon^{2}+|d| \varepsilon}$. Hence,

$$
\delta^{\prime}(\varepsilon)=\frac{2 \varepsilon+|d|}{\sqrt{\varepsilon^{2}+|d| \varepsilon}} \text {, which implies } \delta_{+}^{\prime}(0)=\infty .
$$


It is obvious that $\nu(\lambda)=2$. Let us remark that

$$
\lim _{\varepsilon \rightarrow 0^{+}} \frac{\delta(\varepsilon)}{\varepsilon^{1 / 2}}=2 \sqrt{|d|}
$$

Now, let us see that $c:=\operatorname{cond}_{1 / 2}\left(J_{2}(\lambda, d), \lambda\right)=\sqrt{|d|}$. We need some previous considerations. For a general matrix $A \in \mathbb{C}^{n \times n}$, let $\Lambda(A)=\left\{\lambda_{1}, \ldots, \lambda_{r}\right\}$. Let

$$
A=\sum_{j=1}^{r}\left(\lambda_{j} P_{j}+N_{j}\right)
$$

be the Jordan decomposition of $A$, where for each $j \in\{1, \ldots, r\}, P_{j}$ is the Riesz projector onto the root subspace (or generalized eigenspace) $\mathcal{R}_{\lambda_{j}}(A)$ of $\lambda_{j}$ and along the sum of root subspaces associated with all eigenvalues of $A$ different from $\lambda_{j}$; and $N_{j}:=\left(A-\lambda_{j} I_{n}\right) P_{j}$ is the nilpotent matrix corresponding to $\lambda_{j}$. By $[6$, Theorem 5.4.4 (viii), p. 78], if $\nu_{j}:=\nu\left(\lambda_{j}\right)>1$, then

$$
\operatorname{cond}_{1 / \nu_{j}}\left(A, \lambda_{j}\right)=\left\|N_{j}^{\nu_{j}-1}\right\|^{1 / \nu_{j}} .
$$

In our present example, $A=J_{2}(\lambda, d)=\lambda I_{2}+N$, where

$$
N=\left[\begin{array}{ll}
0 & d \\
0 & 0
\end{array}\right] \text {, }
$$

and thus, $c=\|N\|^{1 / 2}=\sqrt{|d|}$. From $(2.1)$, we have

$$
\lim _{\varepsilon \rightarrow 0^{+}} \frac{\delta(\varepsilon)}{\varepsilon^{1 / 2}}=2 c .
$$

The area $a(\varepsilon)$ is given by $\pi\left(\varepsilon^{2}+|d| \varepsilon\right)$. So, $a^{\prime}(\varepsilon)=\pi(2 \varepsilon+|d|)$; hence, $a_{+}^{\prime}(0)=\pi|d|=$ $\pi c^{2}$. This concludes Example 2.2.

Before discussing the third example, we need to introduce a result on the pseudospectra of nilpotent matrices of nilpotency index two. Here on, we denote by $O_{k}$ the $k \times k$ zero matrix.

Proposition 2.3 ([5], Theorem 3). Let us assume that $q, r$ are nonnegative integers such that $n=2 q+r$. Let $N \in \mathbb{C}^{n \times n}$ be a matrix such that $N^{2}=O_{n}$, whose nonzero singular values are $\sigma_{1}(N) \geq \cdots \geq \sigma_{q}(N)$. Then there exists a unitary matrix $U \in \mathbb{C}^{n \times n}$ such that

$$
U^{*} N U=\left[\begin{array}{cc}
0 & \sigma_{1}(N) \\
0 & 0
\end{array}\right] \oplus \cdots \oplus\left[\begin{array}{cc}
0 & \sigma_{q}(N) \\
0 & 0
\end{array}\right] \oplus O_{r} .
$$

By Example 2.2, or [4, Proposition 2.1] by Cui et al., the $\varepsilon$-pseudospectrum of

$$
\left[\begin{array}{cc}
0 & \sigma_{i}(N) \\
0 & 0
\end{array}\right]
$$


is $\mathcal{D}\left(0, \sqrt{\varepsilon^{2}+\varepsilon \sigma_{i}(N)}\right)$ for $i=1, \ldots, q$. So, by [6, Propositions 5.2.3 and 5.2.4], we have the following result.

Proposition 2.4. Under the hypotheses of Proposition 2.3 for $\varepsilon \geq 0$,

$$
\begin{aligned}
\Lambda_{\varepsilon}(N) & =\bigcup_{i=1}^{q} \mathcal{D}\left(0, \sqrt{\varepsilon^{2}+\varepsilon \sigma_{i}(N)}\right) \cup \mathcal{D}(0, \varepsilon) \\
& =\mathcal{D}\left(0, \sqrt{\varepsilon^{2}+\varepsilon \sigma_{1}(N)}\right) .
\end{aligned}
$$

We will also need that for any $\alpha \in \mathbb{C}, A \in \mathbb{C}^{n \times n}$ and $\varepsilon \geq 0$,

$$
\Lambda_{\varepsilon}\left(\alpha I_{n}+A\right)=\alpha+\Lambda_{\varepsilon}(A) .
$$

ExAmple 2.5. This example is a small generalization of Example 2.2. Let $A$ be any $n$-by- $n$ complex matrix with a unique eigenvalue $\lambda$. Moreover, let us assume that $\nu(\lambda)=2$. Let $N:=A-\lambda I_{n}$; thus, $A=\lambda I_{n}+N$ is the Jordan decomposition of $A$. Hence, by $(2.2), c=\operatorname{cond}_{1 / 2}(A, \lambda)=\|N\|^{1 / 2}=\sqrt{\sigma_{1}\left(A-\lambda I_{n}\right)}$. By Proposition 2.4 and (2.3) we see that for $\varepsilon \geq 0$,

$$
\Lambda_{\varepsilon}(A)=\mathcal{D}\left(\lambda, \sqrt{\varepsilon^{2}+\varepsilon \sigma_{1}\left(A-\lambda I_{n}\right)}\right) .
$$

So, $\delta(\varepsilon)=2 \sqrt{\varepsilon^{2}+\varepsilon \sigma_{1}\left(A-\lambda I_{n}\right)}, a(\varepsilon)=\pi\left(\varepsilon^{2}+\varepsilon \sigma_{1}\left(A-\lambda I_{n}\right)\right)$. Therefore,

$$
\delta^{\prime}(\varepsilon)=\frac{2 \varepsilon+\sigma_{1}\left(A-\lambda I_{n}\right)}{\sqrt{\varepsilon^{2}+\varepsilon \sigma_{1}\left(A-\lambda I_{n}\right)}}, \text { which implies } \delta_{+}^{\prime}(0)=\infty,
$$

and

$$
a^{\prime}(\varepsilon)=\pi\left(2 \varepsilon+\sigma_{1}\left(A-\lambda I_{n}\right)\right), \text { which implies } a_{+}^{\prime}(0)=\pi \sigma_{1}\left(A-\lambda I_{n}\right)=\pi c^{2} .
$$

ExAmple 2.6. Let $\lambda_{1}, \lambda_{2}$ be two different complex numbers. Let $A \in \mathbb{C}^{2 \times 2}$ whose eigenvalues are $\lambda_{1}$ and $\lambda_{2}$. Let us define

$$
d(A):=\sqrt{\operatorname{tr}\left(A^{*} A\right)-\left|\lambda_{1}\right|^{2}-\left|\lambda_{2}\right|^{2}} .
$$

The number $d(A)$ is the departure from normality of $A$. In [6, Proposition 5.5.3, p. 80] was proved that for each $\varepsilon>0$,

$$
\Lambda_{\varepsilon}(A)=\mathcal{D}\left(\lambda_{1}, \varepsilon\right) \cup \mathcal{D}\left(\lambda_{2}, \varepsilon\right) \cup \mathcal{M}_{\lambda_{1}, \lambda_{2}}(d(A), \varepsilon)
$$

where

(2.5) $\mathcal{M}_{\lambda_{1}, \lambda_{2}}(d, \varepsilon):=\left\{z \in \mathbb{C}:\left(\left|z-\lambda_{1}\right|^{2}-\varepsilon^{2}\right)\left(\left|z-\lambda_{2}\right|^{2}-\varepsilon^{2}\right) \leq \varepsilon^{2} d^{2}\right\}, \quad d \geq 0$. 
Let us consider Figure 2.1 that shows the $\varepsilon$-pseudospectrum for the values of $\varepsilon=1.00,1.50,1.75$ of the matrix

$$
A_{1}:=\left[\begin{array}{cc}
1+2 \mathrm{i} & 3 \\
0 & -1-3 \mathrm{i}
\end{array}\right] \text {. }
$$

So, $\lambda_{1}=1+2 \mathrm{i}, \lambda_{2}=-1-3 \mathrm{i},\left\|A_{1}\right\|_{\mathrm{F}}=\sqrt{|1+2 \mathrm{i}|^{2}+3^{2}+|-1-3 \mathrm{i}|^{2}}=\sqrt{5+9+10}=$ $\sqrt{24}$. Thus, $d\left(A_{1}\right)=\sqrt{24-5-10}=\sqrt{9}=3$.

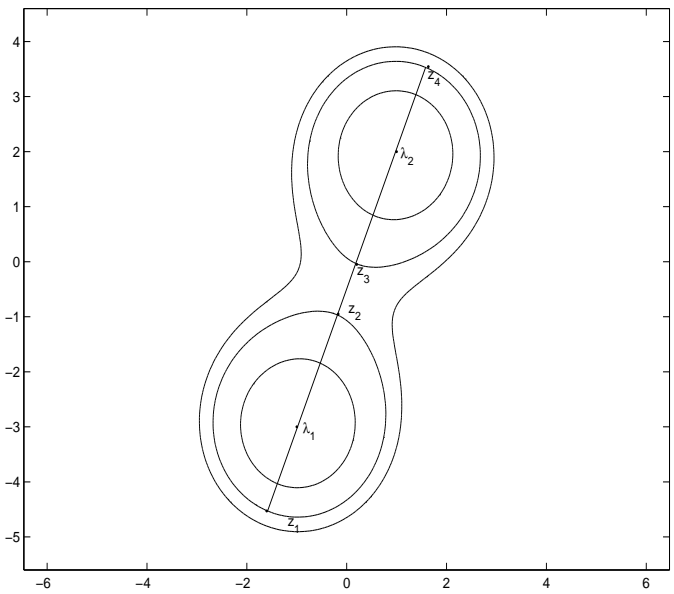

FIG. 2.1. Pseudospectra of $A_{1}$ for $\varepsilon=1.00,1.50,1.75$.

We see that for sufficiently small values of $\varepsilon$ the $\varepsilon$-pseudospectrum of $A_{1}$ has two connected components. Let us fix our attention on $\varepsilon=1.50$. The figure contains a straight line that passes by $\lambda_{1}$ and $\lambda_{2}$. This line intersects the boundary $\partial \Lambda_{\varepsilon}\left(A_{1}\right)$ at the points $z_{1}, z_{2}, z_{3}, z_{4}$. If we consider the eigenvalue $\lambda_{2}$, the diameter $\delta(\varepsilon)$ of the connected component $\mathcal{K}_{\lambda_{2}}(\varepsilon)$ is equal to the distance between $z_{3}$ and $z_{4}$. From $(2.4)$ and $(2.5)$, we can deduce that

$$
\delta(\varepsilon)=\sqrt{1+\varepsilon^{2}+\sqrt{7} \varepsilon}-\sqrt{1+\varepsilon^{2}-\sqrt{7} \varepsilon}
$$

Instead of making a special reasoning for the matrix $A_{1}$, we are going to find a general expression for the diameter $\delta(\varepsilon)$ of $\mathcal{K}_{\lambda_{2}}(\varepsilon)$ for any matrix $A \in \mathbb{C}^{2 \times 2}$ with eigenvalues $\lambda_{1}$ and $\lambda_{2}$. By (2.5), the boundary $\partial \Lambda_{\varepsilon}(A)$ is formed by quasi-Cassini ovals with foci the points $\lambda_{1}$ and $\lambda_{2}$. In fact, the set $\Lambda_{\varepsilon}(A)$ is symmetric about the straight line that joints $\lambda_{1}$ and $\lambda_{2}$. This is a consequence of (2.4) and (2.5). Moreover, $\delta(\varepsilon)=\left|z_{4}-z_{3}\right|=\left|z_{2}-z_{1}\right|$. Later we will need the condition numbers of $\lambda_{1}$ and $\lambda_{2}$ of 
order 1 . These numbers are equal. In fact, by [6, Proposition 5.5.8, p. 83], we have

$$
\operatorname{cond}_{1}\left(A, \lambda_{k}\right)=\sqrt{1+\left(\frac{d(A)}{\left|\lambda_{2}-\lambda_{1}\right|}\right)^{2}}, \quad k=1,2 .
$$

Thus, let $\varepsilon \geq 0$ be such that $\Lambda_{\varepsilon}(A)$ has two connected components. Now, we compute the intersection points $z_{1}, z_{2}, z_{3}, z_{4}$ of the line $z(t):=(1-t) \lambda_{1}+t \lambda_{2}, t \in \mathbb{R}$ with the curve $\partial \Lambda_{\varepsilon}(A)$. By $(2.5)$, this curve is given by the equation

$$
\left(\left|z-\lambda_{1}\right|^{2}-\varepsilon^{2}\right)\left(\left|z-\lambda_{2}\right|^{2}-\varepsilon^{2}\right)-\varepsilon^{2} d(A)^{2}=0
$$

For determining the values of the parameter $t$ that correspond to the points $z_{1}, z_{2}, z_{3}$ and $z_{4}$ we substitute $z(t)$ into $(2.7)$,

$$
\begin{array}{r}
\left|\lambda_{1}-\lambda_{2}\right|^{4} t^{4}-2\left|\lambda_{1}-\lambda_{2}\right|^{4} t^{3}+\left(\left|\lambda_{1}-\lambda_{2}\right|^{4}-2 \varepsilon^{2}\left|\lambda_{1}-\lambda_{2}\right|^{2}\right) t^{2} \\
+2 \varepsilon^{2}\left|\lambda_{1}-\lambda_{2}\right|^{2} t-\varepsilon^{2}\left|\lambda_{1}-\lambda_{2}\right|^{2}+\varepsilon^{4}-\varepsilon^{2} d(A)^{2}=0 .
\end{array}
$$

For simplicity, we write $\theta:=\left|\lambda_{1}-\lambda_{2}\right|$,

$$
\theta^{4} t^{4}-2 \theta^{4} t^{3}+\left(\theta^{4}-2 \varepsilon^{2} \theta^{2}\right) t^{2}+2 \varepsilon^{2} \theta^{2} t-\varepsilon^{2} \theta^{2}+\varepsilon^{4}-\varepsilon^{2} d(A)^{2}=0 .
$$

With the command solve of MAPLE 13 we find the roots of this equation in $t$ obtaining

$$
\begin{aligned}
& t_{1}=\frac{1}{2}-\frac{1}{2 \theta} \sqrt{\theta^{2}+4 \varepsilon^{2}+4 \varepsilon \sqrt{\theta^{2}+d(A)^{2}}}, \\
& t_{2}=\frac{1}{2}-\frac{1}{2 \theta} \sqrt{\theta^{2}+4 \varepsilon^{2}-4 \varepsilon \sqrt{\theta^{2}+d(A)^{2}}}, \\
& t_{3}=\frac{1}{2}+\frac{1}{2 \theta} \sqrt{\theta^{2}+4 \varepsilon^{2}-4 \varepsilon \sqrt{\theta^{2}+d(A)^{2}}}, \\
& t_{4}=\frac{1}{2}+\frac{1}{2 \theta} \sqrt{\theta^{2}+4 \varepsilon^{2}+4 \varepsilon \sqrt{\theta^{2}+d(A)^{2}}},
\end{aligned}
$$

where $t_{1}<0<t_{2}<\frac{1}{2}<t_{3}<1<t_{4}$. Hence, $z_{3}=z\left(t_{3}\right)$ and $z_{4}=z\left(t_{4}\right)$. So,

$$
\delta(\varepsilon)=\left|z_{4}-z_{3}\right|=\frac{1}{2} \sqrt{\theta^{2}+4 \varepsilon^{2}+4 \varepsilon \sqrt{\theta^{2}+d(A)^{2}}}-\frac{1}{2} \sqrt{\theta^{2}+4 \varepsilon^{2}-4 \varepsilon \sqrt{\theta^{2}+d(A)^{2}}} .
$$

Therefore,

$$
\begin{gathered}
\delta^{\prime}(\varepsilon)=\frac{2 \varepsilon+\sqrt{\theta^{2}+d(A)^{2}}}{\sqrt{\theta^{2}+4 \varepsilon^{2}+4 \varepsilon \sqrt{\theta^{2}+d(A)^{2}}}}-\frac{2 \varepsilon-\sqrt{\theta^{2}+d(A)^{2}}}{\sqrt{\theta^{2}+4 \varepsilon^{2}-4 \varepsilon \sqrt{\theta^{2}+d(A)^{2}}}}, \\
\delta_{+}^{\prime}(0)=\frac{2 \sqrt{\theta^{2}+d(A)^{2}}}{\sqrt{\theta^{2}}}=2 \sqrt{1+\left(\frac{d(A)}{\theta}\right)^{2}} .
\end{gathered}
$$


By (2.6), we have $\delta_{+}^{\prime}(0)=2 \operatorname{cond}_{1}\left(A, \lambda_{2}\right)$.

REMARK 2.7. As discussed in the introduction, we will see that the results in these examples are not casual. In fact, we will prove that the condition number of $\lambda$ of order $1 / \nu$ is related with the functions $\delta$ and $a$.

3. Bounds by closed disks. From Theorems 2.6 .6 and 5.4.4 of Karow [6], we infer the following theorem.

TheOREm 3.1. Let $\lambda$ be an eigenvalue of $A \in \mathbb{C}^{n \times n}$ of index $\nu$. For each $\eta \in(0,1]$ there exists an $\varepsilon_{\eta}>0$ such that for every $\varepsilon \in\left(0, \varepsilon_{\eta}\right]$,

$$
\mathcal{D}\left(\lambda,((1-\eta) \varepsilon)^{1 / \nu} c\right) \subset \mathcal{K}_{\lambda}(\varepsilon) \subset \mathcal{D}\left(\lambda,((1+\eta) \varepsilon)^{1 / \nu} c\right)
$$

$c$ being the condition number of $\lambda$ of order $1 / \nu$.

From (3.1), we deduce that

$$
\mathcal{D}\left(0,((1-\eta) \varepsilon)^{1 / \nu} c\right) \subset \mathcal{K}_{\lambda}(\varepsilon)-\lambda \subset \mathcal{D}\left(0,((1+\eta) \varepsilon)^{1 / \nu} c\right) .
$$

Considering the homothecy

$$
z \mapsto \frac{z}{\varepsilon^{1 / \nu}}, \quad z \in \mathbb{C},
$$

we see

$$
\mathcal{D}\left(0,(1-\eta)^{1 / \nu} c\right) \subset \frac{\mathcal{K}_{\lambda}(\varepsilon)-\lambda}{\varepsilon^{1 / \nu}} \subset \mathcal{D}\left(0,(1+\eta)^{1 / \nu} c\right) .
$$

4. Bounds by diameters. Since the diameter function is monotone increasing with respect to $\subset$, by (3.2),

$$
2 c(1-\eta)^{1 / \nu} \leq \frac{\delta(\varepsilon)}{\varepsilon^{1 / \nu}} \leq 2 c(1+\eta)^{1 / \nu} .
$$

Thus, we arrive at the following theorem.

THEOREM 4.1. Let $\nu$ be the index of $\lambda$, and let $c$ be the condition number of $\lambda$ of order $1 / \nu$. Then

$$
\lim _{\varepsilon \rightarrow 0^{+}} \frac{\delta(\varepsilon)}{\varepsilon^{1 / \nu}}=2 c .
$$

Proof. Let us define the functions

$$
\begin{aligned}
& f_{1}(\eta):=1-(1-\eta)^{1 / \nu} \\
& f_{2}(\eta):=(1+\eta)^{1 / \nu}-1,
\end{aligned}
$$


where $\eta \in[0,1]$. If $\nu=1$, then

$$
\begin{aligned}
& f_{1}(\eta):=1-1+\eta=\eta \\
& f_{2}(\eta):=1+\eta-1=\eta
\end{aligned}
$$

so, $f_{1}(\eta)=f_{2}(\eta)$ on $[0,1]$.

If $\nu \geq 2$, we will deduce that $f_{2}(\eta) \leq f_{1}(\eta)$. This last inequality is equivalent to

$$
\begin{aligned}
& (1+\eta)^{1 / \nu}-1 \leq 1-(1-\eta)^{1 / \nu}, \forall \eta \in[0,1] \\
\Longleftrightarrow & (1+\eta)^{1 / \nu}+(1-\eta)^{1 / \nu} \leq 2, \forall \eta \in[0,1] .
\end{aligned}
$$

Let us define

$$
g(\eta):=(1+\eta)^{1 / \nu}+(1-\eta)^{1 / \nu}, \quad \eta \in[0,1]
$$

Then

$$
g^{\prime}(\eta)=\frac{1}{\nu}\left[(1+\eta)^{1 / \nu-1}-(1-\eta)^{1 / \nu-1}\right] .
$$

Since $x^{\alpha}:=\mathrm{e}^{\alpha \ln x}$, when $\alpha<0$ the function $x \mapsto x^{\alpha}$ is decreasing in $(0, \infty)$; therefore, $x \mapsto x^{1 / \nu-1}$ is decreasing in $(0, \infty)$. Hence, if $0<\eta<1$, we see that $1-\eta<1+\eta$; what implies

$$
(1-\eta)^{1 / \nu-1}>(1+\eta)^{1 / \nu-1}
$$

Thus, $g^{\prime}(\eta)<0$. So, $g$ is decreasing on $[0,1]$. Therefore, $g(\eta)<g(0)=2$. That is, $f_{2}(\eta) \leq f_{1}(\eta)$.

Given that $2 c-2 c f_{1}(\eta)=2 c(1-\eta)^{1 / \nu}$ and $2 c+2 c f_{2}(\eta)=2 c(1+\eta)^{1 / \nu}$, by (4.1), we have

$$
2 c-2 c f_{1}(\eta) \leq \frac{\delta(\varepsilon)}{\varepsilon^{1 / \nu}} \leq 2 c+2 c f_{2}(\eta) .
$$

For every $\nu \geq 1, f_{2}(\eta) \leq f_{1}(\eta)$ for $\eta \in[0,1]$. Then,

$$
\begin{gathered}
2 c-2 c f_{1}(\eta) \leq \frac{\delta(\varepsilon)}{\varepsilon^{1 / \nu}} \leq 2 c+2 c f_{1}(\eta) \\
\Longleftrightarrow \quad-2 c f_{1}(\eta) \leq \frac{\delta(\varepsilon)}{\varepsilon^{1 / \nu}}-2 c \leq 2 c f_{1}(\eta),
\end{gathered}
$$

or

$$
\left|\frac{\delta(\varepsilon)}{\varepsilon^{1 / \nu}}-2 c\right| \leq 2 c f_{1}(\eta)
$$


But $\lim _{\eta \rightarrow 0^{+}} f_{1}(\eta)=0$ and $f_{1}(\eta)>0$ for $\eta>0$. Thus, for a fixed $\eta_{0}>0$, there exists an $\eta_{1}>0$ such that $2 c f_{1}\left(\eta_{1}\right)<\eta_{0}$. For this $\eta_{1}$, there is an $\varepsilon_{\eta_{1}}>0$ such that for all $\varepsilon \in\left(0, \varepsilon_{\eta_{1}}\right)$,

$$
\left|\frac{\delta(\varepsilon)}{\varepsilon^{1 / \nu}}-2 c\right| \leq 2 c f_{1}(\eta)<\eta_{0} .
$$

So, there exists the limit

$$
\lim _{\varepsilon \rightarrow 0^{+}} \frac{\delta(\varepsilon)}{\varepsilon^{1 / \nu}}
$$

and it is equal to $2 c$.

5. Derivatives of the diameter. In this section, we relate the right-derivative of the diameter $\delta$ at 0 with the condition number of the eigenvalue $\lambda$, when $\nu=1$. First, we have the following lemma.

Lemma 5.1. Let $A \in \mathbb{C}^{n \times n}$ and $\lambda$ be an eigenvalue of $A$ of index $\nu$. Let $\delta(\varepsilon)$ be the diameter of the connected component of $\Lambda_{\varepsilon}(A)$ that contains $\lambda$. Let $c$ be the condition number of $\lambda$ of order $1 / \nu$. Then, there exists the limit

$$
\lim _{\varepsilon \rightarrow 0^{+}} \frac{\delta(\varepsilon)}{\varepsilon}
$$

and it is equal to

$$
\begin{cases}2 c & \text { if } \quad \nu=1 \\ \infty & \text { if } \quad \nu \geq 2\end{cases}
$$

Proof. If $\nu=1$, Theorem 4.1 implies

$$
\lim _{\varepsilon \rightarrow 0^{+}} \frac{\delta(\varepsilon)}{\varepsilon}=2 c .
$$

If $\nu \geq 2$, from the same Theorem,

$$
\frac{\delta(\varepsilon)}{\varepsilon}=\frac{\delta(\varepsilon)}{\varepsilon^{1 / \nu}} \frac{1}{\varepsilon^{1-1 / \nu}} \rightarrow 2 c \cdot \infty,
$$

when $\varepsilon \rightarrow 0^{+}$.

From this lemma, the next theorem follows immediately.

Theorem 5.2. Let $A \in \mathbb{C}^{n \times n}$ and $\lambda$ be an eigenvalue of $A$ of index $\nu$. Let $\delta(\varepsilon)$ be the diameter of the connected component of $\Lambda_{\varepsilon}(A)$ that contains $\lambda$. Let $c$ be the condition number of $\lambda$ of order $1 / \nu$. Then,

$$
\lim _{\varepsilon \rightarrow 0^{+}} \frac{\delta(\varepsilon)}{\varepsilon}=\left\{\begin{array}{lll}
2 c & \text { if } & \nu=1 \\
\infty & \text { if } & \nu \geq 2
\end{array}\right.
$$

Therefore, $\delta_{+}^{\prime}(0)=2 c$ if $\nu=1$. 
6. Bounds by areas. Since the area function is monotone increasing with respect to $\subset$, by $(3.2)$,

$$
\pi c^{2}(1-\eta)^{2 / \nu} \leq \frac{a(\varepsilon)}{\varepsilon^{2 / \nu}} \leq \pi c^{2}(1+\eta)^{2 / \nu}
$$

where $a(\varepsilon):=$ area or Lebesgue measure of $\mathcal{K}_{\lambda}(\varepsilon)$. Thus, we arrive at the following theorem.

THEOREM 6.1. Let $\nu$ be the index of $\lambda$, and let $c$ be the condition number of $\lambda$ of order $1 / \nu$. Then

$$
\lim _{\varepsilon \rightarrow 0^{+}} \frac{a(\varepsilon)}{\varepsilon^{2 / \nu}}=\pi c^{2} .
$$

Proof. Let us define the functions

$$
\begin{aligned}
& \varphi_{1}(\eta):=1-(1-\eta)^{2 / \nu} \\
& \varphi_{2}(\eta):=(1+\eta)^{2 / \nu}-1 .
\end{aligned}
$$

If $\nu=1$, then

$$
\begin{aligned}
& \varphi_{1}(\eta)=1-(1-\eta)^{2}=2 \eta-\eta^{2}, \\
& \varphi_{2}(\eta)=(1+\eta)^{2}-1=2 \eta+\eta^{2}
\end{aligned}
$$

it is obvious that $\varphi_{1}(\eta) \leq \varphi_{2}(\eta)$ when $0 \leq \eta$.

If $\nu=2$, then

$$
\varphi_{1}(\eta)=1-(1-\eta)=\eta=(1+\eta)-1=\varphi_{2}(\eta)
$$

If $\nu \geq 3$, we will see that

$$
\varphi_{2}(\eta) \leq \varphi_{1}(\eta), \quad \eta \in[0,1]
$$

or, equivalently,

$$
\begin{aligned}
& (1+\eta)^{2 / \nu}-1 \leq 1-(1-\eta)^{2 / \nu}, \quad \eta \in[0,1] \\
& \Longleftrightarrow \quad(1+\eta)^{2 / \nu}+(1-\eta)^{2 / \nu} \leq 2, \quad \eta \in[0,1] .
\end{aligned}
$$

Let us define

$$
\psi(\eta):=(1+\eta)^{2 / \nu}+(1-\eta)^{2 / \nu}, \quad \eta \in[0,1] .
$$


Then

$$
\psi^{\prime}(\eta)=\frac{2}{\nu}\left[(1+\eta)^{2 / \nu-1}-(1-\eta)^{2 / \nu-1}\right] .
$$

As the function $x \mapsto x^{2 / \nu-1}$ is decreasing in $(0, \infty)$, if $0<\eta<1$, then $1-\eta<1+\eta$ implies $(1-\eta)^{2 / \nu-1}>(1+\eta)^{2 / \nu-1}$ and $\psi^{\prime}(\eta)<0$. In consequence, $\psi$ is decreasing in $[0,1]$. Thus, for $\eta \in(0,1], \psi(\eta)<\psi(0)=2$. Accordingly,

$$
\varphi_{2}(\eta) \leq \varphi_{1}(\eta), \quad \eta \in[0,1] .
$$

By (6.2) and (6.3),

$$
\begin{aligned}
& (1-\eta)^{2 / \nu}=1-\varphi_{1}(\eta) \\
& (1+\eta)^{2 / \nu}=1+\varphi_{2}(\eta)
\end{aligned}
$$

Inequalities (6.1) imply

$$
\pi c^{2}-\pi c^{2} \varphi_{1}(\eta) \leq \frac{a(\varepsilon)}{\varepsilon^{2 / \nu}} \leq \pi c^{2}+\pi c^{2} \varphi_{2}(\eta)
$$

The $\nu \leq 2$ case. Since $\varphi_{1}(\eta) \leq \varphi_{2}(\eta)$ in $[0,1]$

$$
-\pi c^{2} \varphi_{2}(\eta) \leq-\pi c^{2} \varphi_{1}(\eta)
$$

Hence, by (6.4),

$$
\pi c^{2}-\pi c^{2} \varphi_{2}(\eta) \leq \frac{a(\varepsilon)}{\varepsilon^{2 / \nu}} \leq \pi c^{2}+\pi c^{2} \varphi_{2}(\eta)
$$

which is equivalent to

$$
\left|\frac{a(\varepsilon)}{\varepsilon^{2 / \nu}}-\pi c^{2}\right| \leq \pi c^{2} \varphi_{2}(\eta) .
$$

Bearing

$$
\lim _{\eta \rightarrow 0^{+}} \pi c^{2} \varphi_{2}(\eta)=0
$$

in mind, we deduce that for a fixed $\eta_{0}>0$ there exists an $\eta_{1}>0$ such that $\pi c^{2} \varphi_{2}\left(\eta_{1}\right)<$ $\eta_{0}$. For this $\eta_{1}$ there is an $\varepsilon_{\eta_{1}}>0$ such that for $\varepsilon \in\left(0, \varepsilon_{\eta_{1}}\right]$,

$$
\left|\frac{a(\varepsilon)}{\varepsilon^{2 / \nu}}-\pi c^{2}\right| \leq \pi c^{2} \varphi_{2}\left(\eta_{1}\right)<\eta_{0} .
$$

This proves that there exists the limit

$$
\lim _{\varepsilon \rightarrow 0^{+}} \frac{a(\varepsilon)}{\varepsilon^{2 / \nu}}
$$


and it is equal to $\pi c^{2}$.

The $\nu \geq 3$ case. As $\varphi_{2}(\eta) \leq \varphi_{1}(\eta)$ in $[0,1]$, from (6.4) we deduce that

$$
\pi c^{2}-\pi c^{2} \varphi_{1}(\eta) \leq \frac{a(\varepsilon)}{\varepsilon^{2 / \nu}} \leq \pi c^{2}+\pi c^{2} \varphi_{1}(\eta)
$$

and, as $\varphi_{1}(\eta)>0$ if $\eta>0$ and

$$
\lim _{\eta \rightarrow 0^{+}} \varphi_{1}(\eta)=0,
$$

by a reasoning analogous to the former one we infer that there exists the limit

$$
\lim _{\varepsilon \rightarrow 0^{+}} \frac{a(\varepsilon)}{\varepsilon^{2 / \nu}}=\pi c^{2}
$$

7. Derivatives of the area. In this section, we establish the relation between the first and second right-derivatives of $a$ at 0 and the condition number of the eigenvalue $\lambda$ of order $1 / \nu$, when $\nu=1$ or 2 . First, we prove the following lemma.

Lemma 7.1. Let $A \in \mathbb{C}^{n \times n}$ and $\lambda$ be an eigenvalue of $A$ of index $\nu$. Let $a(\varepsilon)$ be the area of the connected component of $\Lambda_{\varepsilon}(A)$ that contains $\lambda$. Let $c$ be the condition number of $\lambda$ of order $1 / \nu$. Then, there exists the limit

$$
\lim _{\varepsilon \rightarrow 0^{+}} \frac{a(\varepsilon)}{\varepsilon}
$$

and it is equal to

$$
\left\{\begin{array}{lll}
0 & \text { if } & \nu=1 \\
\pi c^{2} & \text { if } & \nu=2 \\
\infty & \text { if } & \nu \geq 3
\end{array}\right.
$$

Proof. First, let us suppose that $\nu=1$. Then Theorem 6.1 implies

$$
\lim _{\varepsilon \rightarrow 0^{+}} \frac{a(\varepsilon)}{\varepsilon^{2}}=\pi c^{2} .
$$

Hence,

$$
\lim _{\varepsilon \rightarrow 0^{+}} \frac{a(\varepsilon)}{\varepsilon}=\lim _{\varepsilon \rightarrow 0^{+}} \varepsilon \frac{a(\varepsilon)}{\varepsilon^{2}}=\left(\lim _{\varepsilon \rightarrow 0^{+}} \varepsilon\right)\left(\lim _{\varepsilon \rightarrow 0^{+}} \frac{a(\varepsilon)}{\varepsilon^{2}}\right)=0 \cdot \pi c^{2}=0 .
$$

Second, let us assume now that $\nu=2$. From Theorem 6.1,

$$
\lim _{\varepsilon \rightarrow 0^{+}} \frac{a(\varepsilon)}{\varepsilon}=\pi c^{2} .
$$


Finally, when $\nu \geq 3$, it is obvious, by Theorem 6.1 and

$$
\lim _{\varepsilon \rightarrow 0^{+}} \frac{a(\varepsilon)}{\varepsilon}=\lim _{\varepsilon \rightarrow 0^{+}} \frac{a(\varepsilon)}{\varepsilon^{2 / \nu}} \cdot \frac{1}{\varepsilon^{(\nu-2) / \nu}},
$$

that

$$
\lim _{\varepsilon \rightarrow 0^{+}} \frac{a(\varepsilon)}{\varepsilon}=\infty .
$$

Theorem 7.2. Let $A \in \mathbb{C}^{n \times n}$ and $\lambda$ be an eigenvalue of $A$ of index $\nu$. Let a $(\varepsilon)$ be the area of the connected component $\Lambda_{\varepsilon}(A)$ that contains $\lambda$. Then, there exists the right-derivative of a at $0, a_{+}^{\prime}(0)$, and

$$
a_{+}^{\prime}(0)=\left\{\begin{array}{lll}
0 & \text { if } & \nu=1 \\
\pi c^{2} & \text { if } & \nu=2 \\
\infty & \text { if } & \nu \geq 3
\end{array}\right.
$$

Proof. By Lemma 7.1 we deduce that

if $\nu=1$,

$$
a_{+}^{\prime}(0)=\lim _{\varepsilon \rightarrow 0^{+}} \frac{a(\varepsilon)-a(0)}{\varepsilon}=\lim _{\varepsilon \rightarrow 0^{+}} \frac{a(\varepsilon)}{\varepsilon}=0 ;
$$

if $\nu=2$,

$$
a_{+}^{\prime}(0)=\lim _{\varepsilon \rightarrow 0^{+}} \frac{a(\varepsilon)-a(0)}{\varepsilon}=\pi c^{2} ;
$$

if $\nu \geq 3$,

$$
a_{+}^{\prime}(0)=\lim _{\varepsilon \rightarrow 0^{+}} \frac{a(\varepsilon)-a(0)}{\varepsilon}=\infty .
$$

TheOREM 7.3. Let $A \in \mathbb{C}^{n \times n}$ and $\lambda$ be a semisimple eigenvalue. Let $a(\varepsilon)$ be the area of the connected component $\Lambda_{\varepsilon}(A)$ that contains $\lambda$. Let us assume that there exists $a_{+}^{\prime \prime}(0)$. Then

$$
a_{+}^{\prime \prime}(0)=2 \pi c^{2} .
$$

Proof. Let us define the function

$$
A(\varepsilon):= \begin{cases}a(\varepsilon) & \text { if } \varepsilon \geq 0 \\ a(-\varepsilon) & \text { if } \varepsilon<0 .\end{cases}
$$


By Theorem 7.2, as $\nu=1$, there exists $A^{\prime}(0)$ and $A^{\prime}(0)=0$. If we suppose that there exists the derivative $A^{\prime \prime}(0)$, then

$$
A^{\prime \prime}(0)=\lim _{\varepsilon \rightarrow 0} \frac{A(\varepsilon)-2 A(0)+A(-\varepsilon)}{\varepsilon^{2}} .
$$

But, the existence of this limit does not imply the existence of $A^{\prime \prime}(0)$. See [1, Exercise $5-20$.$] .$

When $\varepsilon>0, A(\varepsilon)=a(\varepsilon), A(-\varepsilon)=a(-(-\varepsilon))=a(\varepsilon) ;$ therefore, $A(\varepsilon)+A(-\varepsilon)=$ $2 a(\varepsilon)$.

When $\varepsilon<0, A(\varepsilon)=a(-\varepsilon), A(-\varepsilon)=a(-\varepsilon)$; hence, $A(\varepsilon)+A(-\varepsilon)=2 a(-\varepsilon)$.

Then, by Theorem 6.1,

$$
\begin{gathered}
\lim _{\varepsilon \rightarrow 0^{+}} \frac{A(\varepsilon)-2 A(0)+A(-\varepsilon)}{\varepsilon^{2}}=\lim _{\varepsilon \rightarrow 0^{+}} \frac{2 a(\varepsilon)}{\varepsilon^{2}}=2 \pi c^{2}, \\
\lim _{\varepsilon \rightarrow 0^{-}} \frac{A(\varepsilon)-2 A(0)+A(-\varepsilon)}{\varepsilon^{2}}=\lim _{\varepsilon \rightarrow 0^{-}} \frac{2 a(-\varepsilon)}{(-\varepsilon)^{2}}=\lim _{\beta \rightarrow 0^{+}} \frac{2 a(\beta)}{\beta^{2}}=2 \pi c^{2} .
\end{gathered}
$$

Consequently, there exists the limit

$$
\lim _{\varepsilon \rightarrow 0} \frac{A(\varepsilon)-2 A(0)+A(-\varepsilon)}{\varepsilon^{2}}
$$

and is equal to $2 \pi c^{2}$. So, $A^{\prime \prime}(0)=2 \pi c^{2}$, and $a_{+}^{\prime \prime}(0)=2 \pi c^{2}$.

8. A conjecture. Let $\lambda$ be a semisimple eigenvalue of a matrix $A \in \mathbb{C}^{n \times n}$. We need the definition of a semialgebraic set $S \subset \mathbb{R}^{n}$ and of a semialgebraic function $f: S \rightarrow \mathbb{R}$. These concepts can be seen in [6, Chapter 3, p. 39]. A classical reference is $[2$, Chapter 2, p. 23].

Conjecture 8.1. The functions $\delta, a:[0, \infty) \rightarrow \mathbb{R}$ are semialgebraic.

The following theorem is proved in [3, Lemma 3.1 (ii)].

TheOREm 8.2 (de l'Hôpital inverse rule). If $f, g:[0, \infty) \rightarrow \mathbb{R}$ are semialgebraic functions, $f(0)=g(0)=0$, and there is an $\varepsilon_{0}>0$ such that $g^{\prime}(\varepsilon)>0$ for $\varepsilon \in\left(0, \varepsilon_{0}\right)$, then

$$
\lim _{\varepsilon \rightarrow 0^{+}} \frac{f(\varepsilon)}{g(\varepsilon)}=\ell \in \mathbb{R} \quad \Longrightarrow \quad \lim _{\varepsilon \rightarrow 0^{+}} \frac{f^{\prime}(\varepsilon)}{g^{\prime}(\varepsilon)}=\ell
$$

If Conjecture 8.1 were true, then

$$
\pi c^{2}=\lim _{\varepsilon \rightarrow 0^{+}} \frac{a(\varepsilon)}{\varepsilon^{2}}=\lim _{\varepsilon \rightarrow 0^{+}} \frac{a^{\prime}(\varepsilon)}{2 \varepsilon}=\lim _{\varepsilon \rightarrow 0^{+}} \frac{a^{\prime \prime}(\varepsilon)}{2} .
$$


Thus, the derivative $a_{+}^{\prime \prime}(0)$ would exist and it would be equal to $2 \pi c^{2}$ because $a_{+}^{\prime \prime}(0)=$ $\lim _{\varepsilon \rightarrow 0^{+}} a^{\prime \prime}(\varepsilon)$.

As the area of a region can be expressed by means of a line integral, and taking into account that a parametric integral is differentiable with respect to the parameter when the integrand is, the derivatives $a^{\prime}(\varepsilon)$ and $a^{\prime \prime}(\varepsilon)$ exist for sufficiently small $\varepsilon>0$.

Acknowledgment. The authors thank the referee for the comments and suggestions that let them improve the first and second versions of the paper.

\section{REFERENCES}

[1] T.M. Apostol. Mathematical Analysis. Addison-Wesley Publishing Company, Inc., Reading, Massachusetts, 1957.

[2] J. Bochnak, M. Coste, and M.F. Roy. Real Algebraic Geometry. Springer-Verlag, Berlin, 1998.

[3] J. Bolte, A. Daniilidis, and A.S. Lewis. Tame functions are semismooth. Math. Program., 117:5-19, 2009

[4] J. Cui, V. Forstall, C.K. Li, and V. Yannello. Properties and preservers of the pseudospectrum. Linear Algebra Appl., to appear, 2011, doi:10.1016/j.laa.2011.03.044.

[5] A. George and K.D. Ikramov. Unitary similarity of matrices with quadratic minimal polynomials. Linear Algebra Appl., 349:11-16, 2002.

[6] M. Karow. Geometry of Spectral Value Sets. Ph.D. Thesis, University of Bremen, 2003. 